\title{
ON UNRAMIFIED SEPARABLE ABELIAN $p$-EXTENSIONS OF FUNCTION FIELDS I
}

\author{
HISASI MORIKAWA
}

1. Let $k$ be an algebraically closed field of characteristic $p>0$. Let $K / k$ be a function field of one variable and $L / K$ be an unramified separable abelian extension of degree $p^{r}$ over $K$. The galois automorphisms $\varepsilon_{1}, \ldots, \varepsilon_{p^{\prime}}$ of $L / K$ are naturally extended to automorphisms $\eta\left(\varepsilon_{1}\right), \ldots, \eta\left(\varepsilon_{p^{n}}\right)^{1)}$ of the jacobian variety $J_{L}$ of $L / k$. If we take a svstem of $p$-adic coordinates on $J_{L}$, we get a representation $\left\{M_{p}\left(\eta\left(\varepsilon_{\nu}\right)\right)\right\}$ of the galois group $G(L / K)$ of $L / K$ over $p$-adic integers.

The aim the present note is to determine the $p$-adic integral representation $\left\{M_{p}\left(\eta\left(\varepsilon_{\nu}\right)\right)\right\}$ for cyclic $L / K$ (as a representation over $p$-adic integers). Use will be made of the results in our previous paper [2].

2. Let $\left\{H_{0}, H_{1}, \ldots, H_{s}\right\}$ be the set of all the subgroup of $G(L / K)$ such that $G(L / K) / H_{i}(i=0,1, \ldots, s)$ are cyclic, where $H_{0}$ means $G(L / K)$. We denote by $L_{I_{i}}$ the subfield of $L$ corresponding to $H_{i}$.

We use the following notations:

$p^{\nu_{i}}$ : the degree of $L_{H_{i}}$ over $K$,

$J_{L_{I I}}:$ the jacobian variety of $L_{1 l} / k$,

$\pi_{L^{\prime} / L^{\prime \prime}}$ : the trace mapping of $J_{L^{\prime}}$ onto $J_{L^{\prime \prime}}$, where $L^{\prime} \supset L^{\prime \prime}$,")

$B_{L^{\prime} / L^{\prime \prime}}$ : the irreducible component of $\pi L^{\prime} / L^{\prime-1}(0)$ containing $\{0\}$,

$A_{L^{\prime} / L^{\prime \prime}}$ : the quotient abelian variety of $J_{L^{\prime}}$ by $B_{L^{\prime} L^{\prime \prime}}$,

$\alpha_{L^{\prime} / L^{\prime \prime}}$ : the natural homomorphism of $J_{L^{\prime}}$ onto $\bar{A}_{L^{\prime} / L^{\prime \prime}}$,

$\bar{\pi}_{L^{\prime} / L^{\prime \prime}}:$ the homomorphism of $\bar{A}_{L^{\prime} / L^{\prime \prime}}$ onto $J_{L^{\prime \prime}}$ such that $\pi_{L^{\prime} / L^{\prime \prime}} \alpha_{L^{\prime} / L^{\prime \prime}}=\pi_{L^{\prime} / L^{\prime \prime}}$,

$\rho_{L^{\prime} / L^{\prime \prime}}$ : the cotrace mapping of $J_{L^{\prime \prime}}$ into $J_{L^{\prime}}$,

$\bar{B}_{L^{\prime} / L^{\prime \prime}}$ : the quotient abelian variety of $J_{L^{\prime}}$ by $\rho_{L^{\prime} / L^{\prime \prime}}\left(J_{L^{\prime \prime}}\right)$,

$1(n)$ : the group consisting of all points $t$ on $A$ such that $n t=0$, where 1 is an abelian variety.

Received May 8, 1958.

1) 2) See 1.2 in [2]. 
3. When the order of $.1(p)$ is $p^{r}$, we say, for the sake of simplicity, that the $p$-dimension of .1 is $\gamma$. We denote by $\gamma(.1)$ the $p$-dimension of $i$.

The next Safarevič's lemma is fundamental for our study:

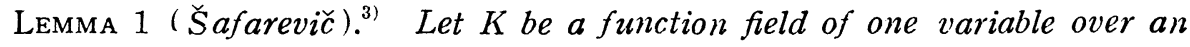
algebraically closed field $k$ and $K^{\prime}$ be a separable normal extension of p-power degree over $K$, where $p$ is the characteristic of $k$. Let $\gamma_{K}$ and $\gamma_{K}$, be respectively the number of independent unramified separable cyclic extensions of degree $p$ over $K$ and $K^{\prime}$. Then we have

$$
\gamma_{K^{\prime}}=\left[K^{\prime}: K\right]\left(\gamma_{K}-1\right)+1 .
$$

On the other hand, the number of independent unramified separable cyclic extensions of degree $p$ over $K$ equals to the $p$-dimension $\gamma\left(J_{K}\right)$ of $J_{K}([2])$. Therefore we get:

LEMMA 2. If $L \supset L_{H_{i}} \supset L_{H_{j}} \supset K$, we have

$$
\begin{aligned}
& \gamma\left(J_{L}\right)=\left[L: L_{H_{i}}\right]\left(\gamma\left(J_{L_{H_{i}}}\right)-1\right)+1=p^{r-\nu_{i}}\left(\gamma\left(J_{H_{i}}\right)-1\right)+1, \\
& \gamma\left(J_{H_{i}}\right)=\left[L_{H_{i}}: L_{I J_{j}}\right]\left(\gamma\left(J_{H_{j}}\right)-1\right)+1=p^{\nu_{i}-v_{j}}\left(\gamma\left(J_{H_{j}}\right)-1\right)+1 .
\end{aligned}
$$

If $x$ is a generic point of $J_{H_{i}}$ over $k$, then $\left(\delta_{J_{I I_{i}}}-\eta\left(\bar{\varepsilon}_{I_{i}}\right)\right) x$ is a generic point of $B_{L_{I_{i}}} / K$ over $k$, where $\delta_{J_{H_{i}}}$ is the identity automorphism of $J_{L_{I_{i}}}$ and $\eta\left(\bar{\varepsilon}_{H_{i}}\right)$ is the extension of a generator $\bar{\varepsilon}_{I I_{i}}$ of the galois group $G\left(L_{H_{i}} / K\right)$ of $L_{I I_{i}} / K$. Therefore we may denote

$$
B_{L_{I I_{i}}} / K=\left(\delta_{J_{L_{I I}}}-\eta\left(\bar{\varepsilon}_{H_{i}}\right)\right)\left(J_{L_{I I_{i}}}\right) .
$$

LeMma 3. $\quad \gamma\left(B_{L_{H_{i}}} / K\right)=\left(\hat{\imath}\left(J_{K}\right)-1\right)\left(\left[L_{H_{i}}: K\right]-1\right)$

$$
=\left(r\left(J_{K}\right)-1\right)\left(p^{\nu_{i}}-1\right)
$$

Proof. $\quad \rho_{L_{1 l_{i}} / K}\left(J_{K}\right)$ and $B_{L_{L I_{i}} / K}$ generate $J_{L_{L_{i}}}$ and $\rho_{L_{H_{i}} / K}\left(J_{K}\right) \cap B_{L_{H_{i}} / K}$ is a finite group. Hence we have

$$
\begin{aligned}
& r\left(B_{L_{I_{i}} / K}\right)=r\left(J_{L_{I I_{i}}} / \rho_{L_{I_{i}} / K}\left(J_{K}\right)\right)=r\left(J_{L_{I I_{i}}}\right)-r\left(\rho_{L_{I I_{i}} / K}\left(J_{K}\right)\right) \\
& =\left(\left[L_{H_{i}}: K\right]\left(\gamma\left(J_{K}\right)-1\right)+1\right)-\gamma\left(J_{K}\right) \\
& =\left(\gamma\left(J_{K}\right)-1\right)\left(\left[L_{I_{i}}: K\right]-1\right)=\left(\gamma\left(J_{K}\right)-1\right)\left(p^{\prime i}-1\right) \text {. }
\end{aligned}
$$

4. First we shall show that $\rho_{L_{i}^{\prime} L_{1 l_{i}}}$ and $\rho_{L_{1 I_{i}} / K}$ are purely inseparable.

3) See $\$ 3$ in $[3]$. 
LEMMA 4. $\rho_{L / L_{H_{i}}}\left(\rho_{L_{H_{i}} / K}\right)$ is purely inseparable.

Proof. Assume that $\rho_{L / L_{H_{i}}}^{-1}(0)\left(\rho_{L_{H_{2}} / K}^{-1}(0)\right)$ constains a $L_{H_{i}}(K)$, non-zero element $t$. Then there exists an element $f$ in $L\left(L_{I I_{i}}\right)$, not in such that $f^{p^{r-\nu_{i}}}\left(f^{p^{\nu}}{ }_{i}\right)$ is contained in $L_{I I_{i}}(K)$. This contradics with the separability of $L / L_{H_{i}}\left(L_{H_{i}} / K\right)$.

Lемма 4. If $G\left(L_{i} / K\right)$ is cyclic, the paddis representation $M_{p}\left(\eta\left(\varepsilon_{\nu}\right)\right)$ is equivalent to the direct sum of $\left(r\left(J_{K}\right)-1\right)$ times of the regular representation and the identical representation as a representation over p-addic numbers.

Proof. We shall prove the proposition by the induction on the degree $p^{r}$ of $L / K$. If $r=0$, the proposition is clearly true. We assume that the proposition is true for the subfield $L_{H}$ such that $L / L_{I I}$ is of degree $p$. Let $\equiv$ be a generator of $G(L / K)$. Then the subgroup $H$ corresponding to $L_{H}$ is $\left(\varepsilon^{p-1}\right)$. Since $\eta(\varepsilon)\left(\rho_{L / L_{H}}\left(J_{L_{H}}\right)\right)=\rho_{L / L_{H H}}\left(J_{L_{H}}\right), \eta(\varepsilon)$ induces an automorphism $\eta^{*}(\varepsilon)$ on $J_{L} / \rho_{L^{\prime} L_{I I}}\left(J_{L_{I}}\right)$. On the other hand $B_{L_{i}^{\prime} L_{I I}}=\left(\delta_{J_{L}}-\eta\left(\varepsilon^{p^{r-1}}\right)\right)\left(J_{L}\right)$ and $J_{L} / \rho_{L / L_{H}}\left(J_{L_{I I}}\right)$ is isogeneous with $B_{L / L_{H}}$, hence $\eta^{*}\left(\varepsilon^{p-1}\right) \neq \delta_{J_{L} / \rho_{L} / L_{I I}}\left(J_{L_{H}}\right)$. By virtue of lemma 3 the $p$-dimension of $B_{L / L_{H}}$ is $(p-1)\left(\gamma\left(J_{L_{H}}\right)-1\right)=(p-1) p^{r-1}\left(\gamma\left(J_{K}\right)-1\right)$. Therefore, since $B_{L / L_{I}}=\left(\delta_{J_{L}}-\eta\left(\varepsilon^{p^{r-1}}\right)\right)\left(J_{L}\right)$, we observe that the $p$-adic representation $\left\{M_{p}\left(\eta^{*}\left(\varepsilon^{\nu}\right)\right)\right\}$ of $\left\{\eta^{*}\left(\varepsilon^{\nu}\right)\right\}$ is equivalent to $\left(\gamma\left(J_{K}\right)-1\right)$-times of the faithful irreducible representation of $G(L / K)$ over $p$-adic integers as a representation over $p$-adic numbers. This shows that $\left\{M_{p}\left(\eta\left(\varepsilon^{\nu}\right)\right)\right\}$ is equivalent to the direct sum of $\left(\gamma\left(J_{K}\right)-1\right)$-times of the regular representation and the identical representation as a representation over $p$-adic numbers.

Proposition 1. The p-adic representation $\left\{M_{\rho}\left(\eta\left(\varepsilon_{0}\right)\right)\right\}$ of $G\left(L^{\prime} K\right)$ is equivalent to the direct sum of $\left(\gamma\left(J_{K}\right)-1\right)$-times of the regular representation and the identical representation as a representation over p-adic numbers.

Proof. Since $G(L / K)$ is abelian, $\left\{M_{p}\left(\eta\left(\varepsilon_{\nu}\right)\right)\right\}$ is equivalent to a direct sum of $p$-adic irreducible representations of cyclic factor groups of $G(L / K)$. By virtue of lemma $4,\left\{M_{p}\left(\eta\left(\varepsilon_{\nu}\right)\right)\right\}$ contains at least $\left(r\left(J_{K}\right)-1\right)$-times of the $p$-adic irreducible faithful representation of any non-trivial cyclic factor group of $G\left(L_{i} / K\right)$. On the other hand the degree $p^{r}\left(\gamma\left(J_{K}\right)-1\right)+1$ of $\left\{M_{p}\left(\eta\left(\varepsilon_{\nu}\right)\right)\right\}$ is equal to that of the direct sum of $\left(\gamma\left(J_{K}\right)-1\right)$-times of the regular representation and the identical representation. Moreover the latter sum contains 
exactly $\left(\gamma\left(J_{K}\right)-1\right)$-times of $p$-adic irreducible faithful representation of any non-trivial cyclic factor group of $G(L / K)$ and $\gamma\left(J_{K}\right)$-times of the identical representation. This shows that $\left\{M_{p}\left(\eta\left(\varepsilon_{\nu}\right)\right)\right\}$ is equivalent to the sum of $\left(\gamma\left(J_{K}\right)-1\right)$-times of regular representation and the identical representation as a representation over $p$-adic numbers.

PROPOSITION 2. $\rho_{L_{H_{i} / K}}\left(J_{K}\right) \cap B_{L_{H_{i}} / K}=\rho_{L_{H_{i}} / K} \pi_{L_{H_{i}} / K}\left(J_{L_{H_{i}}}\left(p^{\nu_{i}}\right)\right)$

$$
=\left(\delta_{J_{B_{H_{i}} / K}}-\eta_{B_{L_{H_{i}} / K}}\left(\bar{\varepsilon}_{H_{i}}\right)\right)^{-1}(0) \text {, }
$$

where $\eta_{B_{L_{H_{i}} / K}}\left(\bar{\varepsilon}_{H_{i}}\right)$ is the restriction of $\eta\left(\bar{\varepsilon}_{H_{i}}\right)$ on $B_{L_{H_{i}} / H}$.

Proof. Since $\pi_{L_{H_{i} / K} / K} \rho_{L_{H_{i}} / K}=\bar{\pi}_{L_{H_{i} / K} / K} \alpha_{L_{H_{i}} / K} \rho_{L_{H_{i}} / K}=p^{\nu_{i}} \delta_{J_{K}}$, we have $\rho_{L_{H_{i}} / K} \bar{\pi}_{L_{H_{i}} / K}$ $\left(\bar{A}_{L_{H_{i} / K}}\left(p^{\nu_{i}}\right)\right)=\alpha_{L_{H_{i} / K}}^{-1}(0) \cap \rho_{L_{H_{i}} / K}\left(J_{K}\right)=B_{L_{H_{i}} / K} \cap \rho_{L_{H_{i}} / K}\left(J_{K}\right)$. On the other hand $\pi_{L_{H_{i}} / K}\left(J_{L_{H_{i}}}\left(p^{\nu_{i}}\right)=\bar{\pi}_{L_{H_{i} / K}}\left(\bar{A}_{L_{H_{i} / K}}\left(p^{\mathrm{i} i}\right)\right)\right.$, hence we have $\rho_{L_{H_{i}} / K}\left(J_{K}\right) \cap B_{L_{H_{i}} / K}=\rho_{L_{H_{i}} / K}$ $\pi_{L_{H_{i}} / K}\left(J_{L_{H_{i}}}\left(p^{\nu_{i}}\right)\right)$.

From $\left(\delta_{J_{L_{H_{i}}}}-\eta\left(\bar{\varepsilon}_{H_{i}}\right)\right) \rho_{L_{H_{i}} / K}\left(J_{K}\right)=0$, we observe that $\rho_{L_{H_{i}} / K}\left(J_{K}\right) \cap B_{L_{H_{i}} / K}$ $C\left(\delta_{B_{L_{i}} / K}-\eta_{B_{L_{H i} / K}}\left(\bar{\varepsilon}_{H_{i}}\right)\right)^{-1}(0)$. Therefore it is sufficient to prove that the order of $\rho_{L_{H_{i}} / K}\left(J_{K}\right) \cap B_{L_{H} / K}$ equals to that of $\left(\varepsilon_{R_{L_{H i}}{ }^{\prime K}}-\eta_{B_{L_{H i} / K}}\left(\bar{\varepsilon}_{H_{i}}\right)\right)^{-1}(0)$. Since $J_{K}\left(p^{\nu}\right) / \rho_{L_{H_{i}} / K} J_{L_{H_{i}}}\left(p^{\nu_{i}}\right) \cong G\left(L_{H_{i}} / K\right)$ and $\rho_{L_{H_{i}} / K}$ is purely inseparable, the order of $\rho_{L_{H_{i}} / K}\left(J_{K}\right) \cap B_{L_{H_{i}} / K}$ is $p^{\left(\gamma\left(J_{K}\right)-1\right) v_{i}}$. On the other hand, by virtue of proposition 1 , the $p$-adic representation $\left\{M_{p}\left(\eta_{B_{L_{H_{i}} / K}}\left(\overline{3}_{H_{i}} \nu\right)\right)\right\}$ of $G\left(L_{H_{i}} / K\right)$ is equivalent to $\left(r\left(J_{K}\right)-1\right)$-times of the sum of all the non-trivial irreducible $p$-adic representations of $G\left(L_{H_{i}} / K\right)$ as a representation over $p$-adic numbers. This shows that the order of $\left(\delta_{J_{B_{L_{H}} / K}}-\eta_{B_{L_{H_{i}} / K}}\left(\bar{\varepsilon}_{H_{i}}\right)\right)^{-1}(0)$ is $p^{v_{i}\left(r\left(J_{K}\right)-1\right)}$. We have proved proposition 2 .

5. Using proposition 2, we shall determine the structure of $J_{L}$.

Proposition 3. Let $H_{j}$ be the subgroup of $H_{i}$ such that $H_{i} / H_{j}$ is a cyclic group of order $p$. Then $\rho_{L / L_{j}}\left(B_{L_{j} / L_{i}}\right)$ is the invariant abelian subvariety for $\left\{\eta\left(\varepsilon_{\nu}\right)\right\}$ on $J_{L}$ such that the $p$ adic representation $\left\{M_{p}^{*}\left(\eta\left(\varepsilon_{\nu}\right)\right)\right\}$ of $\left\{\eta\left(\varepsilon_{\nu}\right)\right\}$ on $\rho_{L / L_{H J}}\left(B_{L_{H J} / L_{H_{i}}}\right)$ is equivalent to $\left(\gamma\left(J_{K}\right)-1\right)$-times of the p-adic irreducible representation $\left\{M_{p}^{H_{i}}\left(\eta\left(\varepsilon_{v}\right)\right)\right\}$ of $G(L / K)$ whose kernel is $H_{j}$, as a representation over p-adic numbers.

Proof. By virtue of proposition 1, the multiplicity of $\left\{M_{p}^{H_{i}}\left(\eta\left(\varepsilon_{0}\right)\right)\right\}$ in 
$\rho_{L / L_{H_{j}}}\left(J_{L_{I I_{j}}}\right)$ is $\left(\gamma\left(J_{K}\right)-1\right)$ and that in $\rho_{L / L_{I I_{i}}}\left(J_{L_{I_{i}}}\right)$ is zero. This proves the proposition.

We denote by $B_{H_{j}}$ the above $\rho_{L / L_{H}}\left(B_{L_{H_{j}} / L_{H_{i}}}\right)$. Namely $B_{I_{j}}$ means the invariant subabelian variety on $J_{L}$ for $\left\{\eta\left(\varepsilon^{\nu}\right)\right\}$ such that the $p$-adic representation of $\left\{h\left(\varepsilon_{v}\right)\right\}$ on $B_{H_{i}}$ is equivalent to $\left(r\left(J_{K}\right)-1\right)$-times of the $p$-adic irreducible representation of $G(L / K)$ whose kernel is $H_{j}$, as a representation over p-adic numbers. Then we get

THEOREM 1. $J_{L}$ is isogeneous with $B_{H_{1}}+\ldots+B_{I_{s}}+\rho_{L / K}\left(J_{K}\right)$ and the subvarieties $B_{H_{1}}, \ldots, B_{H_{s}}$ and $\rho_{L / K}\left(J_{K}\right)$ satisfy the following conditions:

$$
\begin{gathered}
\rho_{L / K}\left(J_{K}\right) \cap B_{H:}=\rho_{L / K} \pi_{L_{H_{i}} / K}\left(J_{H_{l}}(p)\right), \\
\text { if } L_{H_{i}} \cap L_{H_{j}}=L_{H_{l}}
\end{gathered}
$$

then

$$
\begin{aligned}
B_{H_{i}} \cap B_{H_{j}} & =\rho_{L / L_{H_{l}}}\left(\pi_{L_{H_{i} / L_{H_{l}}}}\left(J_{L_{H_{i}}}(p)\right) \cap \pi_{L_{H_{j}} / L_{H_{i}}}\left(J_{L_{\cdot H_{j}}}(p)\right)\right. \\
& =\rho_{L / L_{H_{l}}}\left(J_{L_{H_{l}}}\right) \cap B_{H_{j}} \cap B_{H_{j}} .
\end{aligned}
$$

Proof. The first assertion has been proved in proposition 1 . Let $H_{i}^{\prime}$ be the subgroup of $G(L / K)$ such that $H_{i}^{\prime} / H_{i}$ is a cyclic group of order $p$. Then, by virtue of theorem 2 in [2], we observe that $J_{K}\left(p^{\nu_{i}-1}\right) / \pi_{L_{H_{i}}{ }^{\prime} / K}\left(J_{L_{H_{i}}{ }^{\prime}}\left(p^{\nu_{i}-1}\right)\right)$ $\cong G(L ! K) / H_{i}^{\prime}$ and $J_{K}\left(p^{\prime \prime i}\right) / \pi_{L_{H_{i}} / K}\left(J_{L_{H_{i}}}\left(p^{\prime \prime i}\right)\right) \cong G(L / K) / H_{i}$, hence $J_{K}(p) / \pi_{L_{H_{i}} / K}$ $\left(J_{L_{H_{i}}}(p)\right)$ and $J_{K}(p) / \pi_{L_{H_{i}} / K}\left(J_{L_{H_{i}}}(p)\right)$ are isomorphic. On the other hand $\pi_{L_{H_{i}}{ }^{\prime} / K}\left(J_{L_{H_{i}}}(p)\right) \subseteq \pi_{L_{H_{i}} / K}\left(J_{L_{H_{i}}{ }^{\prime}}(p)\right)$, therefore we have $\pi_{L_{H_{i}}{ }^{\prime} / K} J_{L_{H_{i}}{ }^{\prime}}(p)=\pi_{L_{H_{i}}{ }^{\prime} K}\left(J_{L_{H_{i}}}(p)\right)$ for $L_{I I_{i}} \neq K$. Moreover, since $\left[J_{L_{H_{i}}}(p): \pi_{L_{H_{i}}{ }^{\prime} L_{H_{i}}}\left(J_{L_{H_{i}}}(p)\right)\right]=p$ and $\rho_{L_{H_{i}} / K} \pi_{L_{H_{i}} / K}$ $\left(J_{L_{H_{i}}}(p)\right) \subseteq \rho_{L_{H_{i}}{ }^{\prime} / K}\left(J_{K}(p)\right)$, we have $\rho_{L_{H_{i}} / K^{\prime}} \pi_{L_{H_{i}}{ }^{\prime} / K}\left(J_{L_{H_{i}}}(p)\right)=\rho_{L_{H_{i}}{ }^{\prime} / K} \pi_{L_{H_{i}} / K}\left(J_{L_{H_{i}}}(p)\right)$ $=\rho_{L_{H_{i}} / K}\left(I_{K}\right) \cap \pi_{L_{H_{i}} / L_{H_{i}}{ }^{\prime}}\left(J_{L_{H_{i}}}(p)\right)$.

On the other hand, by virtue of proposition 2 and the pure inseparability of $\rho_{L^{\prime} K}$, we have

$$
\begin{aligned}
\rho_{L / K}^{\prime K}\left(J_{K}\right) \cap B_{H_{i}} & =\rho_{L / K}\left(J_{K}\right) \cap \rho_{L / L_{H_{i}}}\left(B_{L_{H_{i}} / L_{H_{i}}{ }^{\prime}}\right) \\
& =\rho_{L / L_{H_{i}}}\left(\rho_{L_{H_{i}} / K}\left(J_{K}\right) \cap\left(\rho_{L_{H_{i}} / L_{H_{i}}}\left(J_{L_{H_{i}}}\right) \cap B_{L_{H_{i}} / L_{H_{i}}{ }^{\prime}}\right)\right) \\
& =\rho_{L / L_{H_{i}}}\left(\rho_{L_{H_{i}} / K}\left(J_{K}\right) \cap \rho_{L_{H_{i}} / L_{H_{i}}{ }^{\prime}} \pi_{L_{H_{i}} / L_{H_{i}}}\left(J_{L_{H_{i}}}(p)\right)\right. \\
& =\rho_{L / L_{H_{i}}}\left(\rho_{L_{H_{i}} / K}\left(J_{K}\right) \cap \pi_{L_{I_{i}} / L_{I I_{i}}}\left(J_{L_{H_{i}}}(p)\right)\right) .
\end{aligned}
$$


This proves (1).

By virtue of (1) we have

$$
\rho_{L: L_{I_{i}}}\left(J_{L_{I_{i}}}\right) \cap B_{I_{j}}=\rho_{L / L_{I_{i}}} \pi_{L_{I I_{j}} \frown H_{j_{j}^{\prime}} I_{H_{H_{i}}}}\left(J_{L_{I I_{i}} \frown I_{j}}(p)\right)
$$

and

$$
\rho_{L_{i}^{\prime} L I I_{j}}\left(J_{L_{H_{j}}}\right) \cap B_{I I_{i}}=\rho_{L / L_{I I_{j}}} \pi_{L_{I I_{i}} \frown H_{j}^{\prime} I_{I I_{j}}}\left(J_{L_{I I_{i}} \frown H_{j}}(p)\right) .
$$

On the other hand $\rho_{L / L_{I I_{i}}}\left(J_{L_{H_{l}^{\prime}}}\right) \cap \rho_{L^{\prime} L_{I I_{j}}}\left(J_{L_{I I_{j}}}\right) \subset \rho_{L^{\prime} L_{I I_{i}} \frown L_{I I_{j}}}\left(J_{L_{I I_{i}} \frown L_{I H_{j}}}\right)$ hence

$$
\begin{aligned}
B_{I I} \cap B_{H_{j}}= & \rho_{L / L_{I j}} \pi_{L_{H_{i}} \frown I I_{j} / L_{H_{i}}}\left(J_{L_{I I_{i}} \frown H_{j}}(p)\right) \\
& \cap \rho_{L / L_{I_{j}}} \pi_{L_{I I_{i}} \frown H_{j} / L_{H_{j}}}\left(J_{L_{I_{I}} \frown H_{j}}(p)\right) \subset \rho_{L / L_{H_{i}} \frown L_{I_{j}}}\left(J_{L_{H_{t}} \frown L_{I I_{j}}}\right) .
\end{aligned}
$$

This shows that

$$
B_{H H_{i}} \cap B_{I I_{j}}=B_{H_{i}} \cap B_{H_{j}} \cap \varrho_{L / L_{H_{i}}} \cap L_{H H_{j}}\left(J_{L_{H_{i}}} \cap L_{H_{j}}(p)\right) .
$$

Therefore, if $L_{H_{i}} \cap L_{l_{j}}=L_{H_{l}}$, we have

$$
\begin{aligned}
B_{I_{i}} \cap B_{H_{j}} & =\left(\rho_{L / L_{I_{l}}}\left(J_{L_{H_{l}}}\right) \cap B_{H_{i}}\right) \cap\left(\rho_{L / L_{H_{l}}}\left(J_{L_{H_{l}}}\right) \cap B_{H_{j}}\right) \\
& =\rho_{L / L_{H_{l}}}\left(\pi_{L_{H_{i}} / L_{H_{l}}}\left(J_{L_{H_{i}}}(p)\right) \cap \rho_{L_{H_{j}} / L_{H_{l}}}\left(J_{L_{H_{j}}}(p)\right)\right) .
\end{aligned}
$$

6. In this section we shall study some $p$-adic integral representations of cyclic groups.

Lеммa 5. Let $\left\{R\left(\varepsilon^{\prime \prime}\right)\right\}$ be a regular representation and $\left\{M\left(\varepsilon^{\prime \prime}\right)\right\}$ be any representation of a cyclic group $(\varepsilon)$. Then a p-adic integral representation

$$
\left\{\left(\begin{array}{cc}
M\left(\varepsilon^{\nu}\right) & 0 \\
A\left(\varepsilon^{\nu}\right) & R\left(\varepsilon^{\nu}\right)
\end{array}\right)\right\}
$$

is equivalent to

$$
\left\{\left(\begin{array}{cc}
M\left(\varepsilon^{2}\right) & 0 \\
0 & R\left(\varepsilon^{2}\right)
\end{array}\right)\right\}
$$

as a p-adic integral representation.

Since the group ring $Z_{p}[G]$ over $p$-adic integers is projective as a left $G$-module, this lemma is clearly time.

Lemma 6. Let $G=(\varepsilon)$ be a cyclic group of order $p^{r}$ and $G_{r-1}$ be the subgroup $\left(\varepsilon^{p^{r-1}}\right)$. Let $\left\{N\left(\varepsilon^{p^{r-1}}\right)^{2}\right\}$ be the non-trivial irreducible $p$ integral representation of $G_{r-1}$. Let $\left\{\hat{M}(\varepsilon)^{\prime}\right\}$ be the representation of $G$ induced by 
$\left\{N\left(\varepsilon^{p^{r-1}}\right)^{2}\right\}$ and $\left\{R_{r-1}(\bar{\varepsilon})^{\nu}\right\}$ be the regular representation of $G / G_{r-1}$.

Then any $p$-adic integral representation of the following type

is equivalent to

$$
\left\{\left(\begin{array}{cc}
E_{n} \times \hat{M}(\varepsilon) & 0 \\
1(\varepsilon) & E_{n} \times R_{r-1}(\bar{\varepsilon})
\end{array}\right)^{\nu}\right\}
$$

$$
\left\{\left(\begin{array}{ccc}
E_{n} \times \hat{M}(\varepsilon) & 0 \\
\mathfrak{a} & 0 & E_{n} \times R_{r-1}(\bar{\Xi})
\end{array}\right)^{2}\right\}
$$

as a representation over p-adic integers, where $\bar{\varepsilon}$ is the class of $\varepsilon$ in $G / G_{r-1}$ and $a$ is a p-adic integral $\left(n p^{r-1}, n(p-1)\right)$-matrix.

Proof. Since $\left\{\hat{M}(\varepsilon)^{2}\right\}$ is the induced representation of $G$ by $\left\{N\left(\varepsilon^{p^{r-1}}\right)^{2}\right\}$, we have

$$
E_{n} \times \hat{M}(\varepsilon)=\left(\begin{array}{llc}
0 & E_{n(p-1} ; & 0 \ldots 0 \\
0 & 0 & E_{n(p-1)} \\
0 & & E_{n, p-1)} \\
E_{n} \times N & 0 \ldots \ldots
\end{array}\right),
$$

where $N=N\left(\varepsilon^{p^{r-1}}\right)$. We put

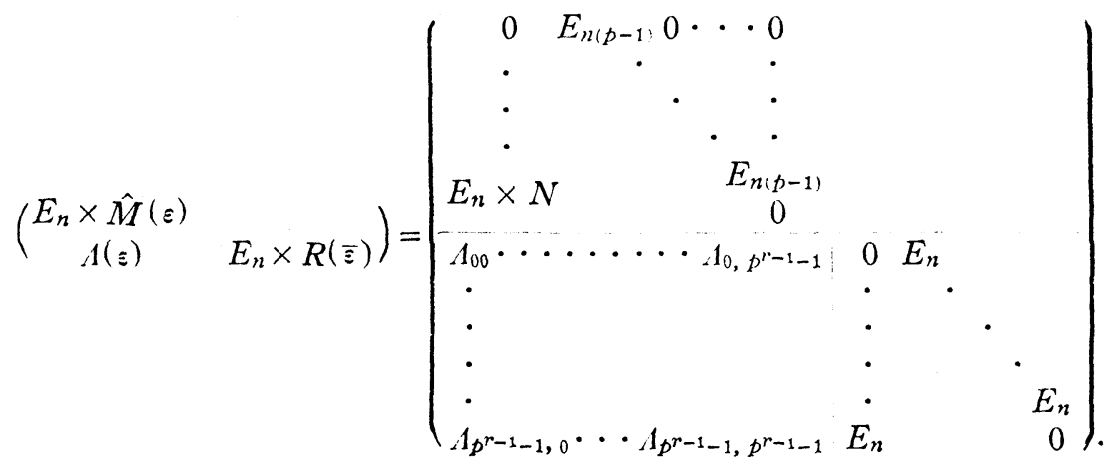

We choose $p$-adic integral matrices $X_{i j}$ such that

$$
X_{i, 0}=0, X_{i+1,1}=\Lambda_{i, 1}, X_{i+1, j}=A_{i, j}-X_{i, j-1}
$$

$\left(i=0,1, \ldots, p^{r-1}-1 ; j=1,2, \ldots, p^{r-1}-1\right)$, where $X_{p^{r-1}, j}=X_{0, j}$ and $X_{i,-1}$ $=X_{i, p^{r-1}-1}$. Then we have

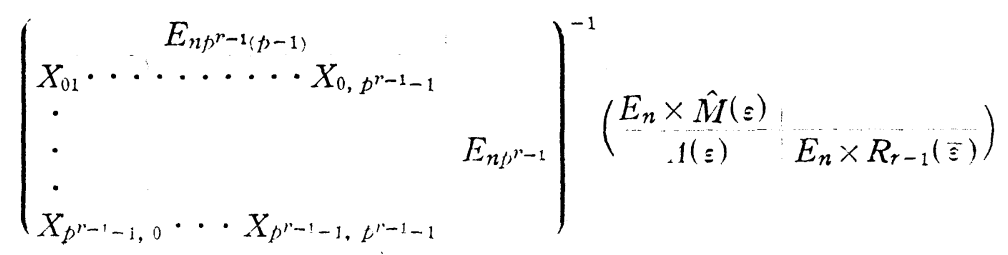




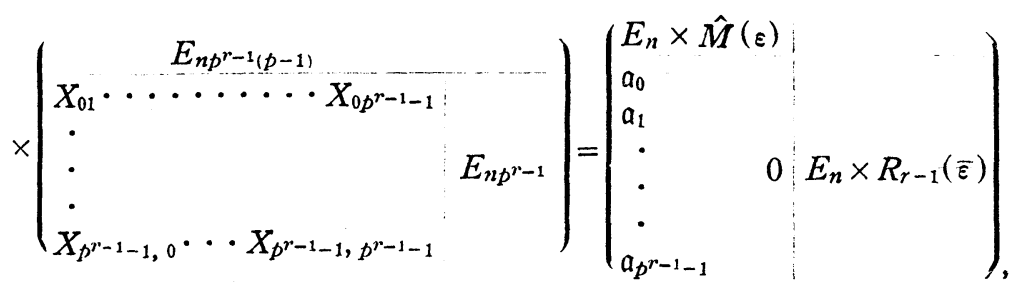

where

$$
\mathfrak{a}_{i}=A_{i, 0}+X_{i+1,0}\left(E_{n} \times N-E_{n(p-1)}\right) .
$$

This proves the lemma.

Leмma 7. In the notations in lemma 6, let

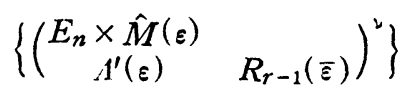

and

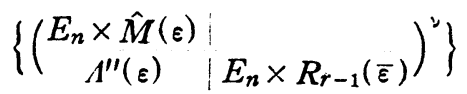

be p-adic integral representations of $(\varepsilon)$ whose restrictions on the subgroup $\left(\varepsilon^{p^{r-1}}\right)$ are equivalent (as p-adic integral representation). Then the representations $(*)$ and $(* *)$ are equivalent (as p-adic integral representations).

Proof. By virtue of lemma 6, we may assume that

$$
A^{\prime}(\varepsilon)=\left(a^{\prime}, 0\right) \text { and } A^{\prime \prime}(\varepsilon)=\left(a^{\prime \prime}, 0\right)
$$

with $p$-adic integral $\left(n p^{r-1}, n(p-1)\right)$-matrices $a^{\prime}$ and $a^{\prime \prime}$. From the assumption of the lemma, we have

$$
\begin{aligned}
& \Lambda^{\prime \prime}\left(\varepsilon^{p^{r-1}}\right)=\Lambda^{\prime}\left(\varepsilon^{p^{r-1}}\right)+E_{n} \times R_{r-1}\left(\varepsilon^{p^{r-1}}\right) X-X \cdot E_{n} \times \hat{M}\left(\varepsilon^{p^{r-1}}\right) \\
& \quad=\Lambda^{\prime}\left(\varepsilon^{p^{r-1}}\right)+X-X\left(\begin{array}{c}
E_{n} \times N\left(\varepsilon^{p^{r-1}}\right) \\
\cdot \\
\cdot \\
E_{n} \times N\left(\varepsilon^{p^{r-1}}\right)
\end{array}\right),
\end{aligned}
$$

with a $p$-adic integral matrix $X$.

On the other hand we observe that

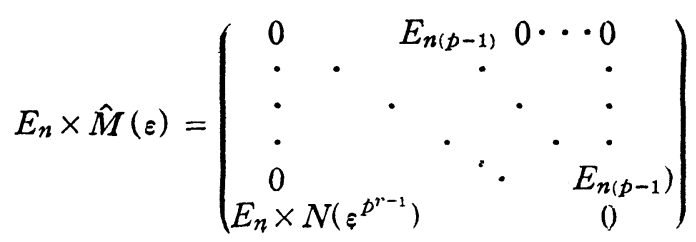


and

$$
\begin{aligned}
& A^{\prime \prime}\left(\varepsilon^{p^{r-1}}\right)-A^{\prime}\left(\varepsilon^{p^{r-1}}\right) \\
& =\sum_{\nu=0}^{p^{r-1}-1} E_{n} \times R_{r-1}(\bar{\varepsilon})^{p^{r-1}-1-\nu}\left(A^{\prime \prime}(\varepsilon)-A^{\prime}(\varepsilon)\right) E_{n} \times \hat{M}(\varepsilon)^{\nu} .
\end{aligned}
$$

Therefore, putting

$$
A^{\prime \prime}\left(\varepsilon^{p^{r-1}}\right)-A^{\prime}\left(\varepsilon^{p^{r-1}}\right)=(\overbrace{P,}^{n(p-1)} \overbrace{Q}^{n\left(p^{p^{r}-1}-1\right)(p-1)}),
$$

we have

$$
P=E_{n} \times R(\bar{\varepsilon})^{p^{r-1}-1}\left(a^{\prime \prime}-a^{\prime}\right) .
$$

On the other hand, if we put

$$
X=(\overbrace{Y,}^{n(p-1)} \overbrace{Z}^{n\left(p^{r-1}-1\right)(p-1)}),
$$

we have

$$
P=Y\left(E_{n(p-1)}-E_{n} \times N\left(\varepsilon^{p^{r-1}}\right)\right) .
$$

This shows that

$$
\begin{aligned}
\mathfrak{a}^{\prime \prime}-\mathfrak{a}^{\prime} & =E_{n} \times R_{r-1}(\bar{\varepsilon})^{1-p^{r-1}} Y\left(E_{n(p-1)}-E_{n} \times N\left(\varepsilon^{p^{r-1}}\right)\right) \\
& =E_{n} \times R_{r-1}(\bar{\varepsilon}) Y\left(E_{n(p-1 ;}-E_{n} \times N\left(\varepsilon^{p^{r-1}}\right)\right)
\end{aligned}
$$

Hence, putting $Y_{1}=E_{n} \times R_{r-1}(\bar{\varepsilon}) Y$, we have

$$
\begin{aligned}
& \left(\frac{E_{n} \times \hat{M}(\varepsilon) \mid}{\Lambda^{\prime \prime}(\varepsilon) \mid E_{n} \times R_{r-1}(\bar{\varepsilon})}\right)=\left(\begin{array}{c}
E_{n(p-1) p^{r-1}} \mid \\
\hline Y_{1} 0 \\
E_{n p^{r-1}}
\end{array}\right)^{-1} \\
& \times\left(\begin{array}{c|c|c}
E_{n} \times \hat{M}(\varepsilon) & \\
\hline \Lambda^{\prime}(\varepsilon) & E_{n} \times R_{r-1}(\bar{\varepsilon})
\end{array}\right)\left(\begin{array}{c|c}
E_{n(p-1) p^{r-1}} \\
\hline Y_{1} 0 & E_{n p^{r-1}}
\end{array}\right) .
\end{aligned}
$$

7. In this section, using proposition 2 and lemma 7, we shall prove the main theorem.

Lemma 8. If $G(L / K)$ is a cyclic group of order $p$, the $p$-adic representation $\left\{M_{p}\left(\eta\left(\varepsilon^{\nu}\right)\right)\right\}$ of the galois group is equivalent to the direct sum of $\left(\gamma\left(J_{K}\right)\right.$ -1)-times of the regular representation and the identical representation as a representation over p-adic integers.

Proof. First we notice that there exist only two inequivalent $p$-adic integral representations of $G(L / K)$ 


$$
\left\{\left(\begin{array}{cc}
1 & \\
\hline 1 \\
0 \\
\cdot \\
\cdot \\
\cdot
\end{array}\right)^{\nu} N(\varepsilon)\right\} \text { and }\left\{\left(\begin{array}{ll}
1 & \\
0 & N(\varepsilon)
\end{array}\right)^{2}\right\}
$$

which have the same irreducible components 1 and $\left\{N(\varepsilon)^{\nu}\right\}$, where $\left\{N(\varepsilon)^{\nu}\right\}$ is the non-trivial irreducible representation."

By virtue of proposition 2, we observe that

$$
\rho_{L / K}\left(J_{K}\right) \cap B_{L / K}=\left(\delta_{B_{L / K}}-r_{i}(\varepsilon)_{B_{L / K}}\right)^{-1}(0) .
$$

This shows that $\left\{M_{p}(\eta(\varepsilon))^{\text {' }}\right\}$ contains no

$$
\left\{\left(\begin{array}{cc}
1 & 0 \\
0 & N(\varepsilon)
\end{array}\right)^{\nu}\right\}
$$

as a component. Namely there exists a system of $p$-adic coordinates on $J_{L}$ such that

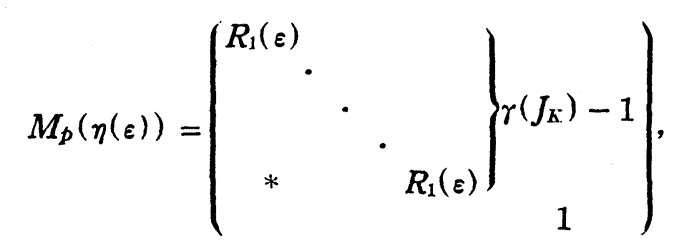

where $\left\{R_{1}(\varepsilon)^{2}\right\}$ is the regular representation of $G(L / K)$. By virtue of lemma 5 , there exists a system of $p$-adic coordinates on $J_{L}$ such that

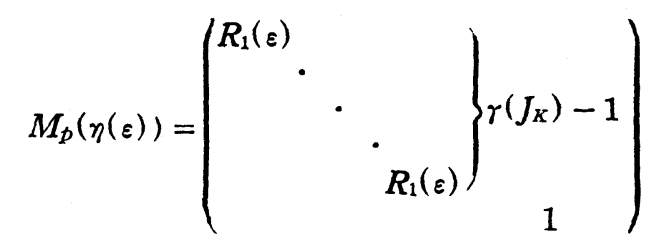

1) 5) Since $\left[Q_{p}\left(p^{r} \sqrt{1}\right): Q_{p}\right]=p^{r-1}(p-1)$ and the class number of $Q_{p}\left({ }^{r} \sqrt{1}\right)$ is one, there exists only one faithful $p$-adic integral irreducible representation. Moreover $G=(\varepsilon)$ is cyclic, hence a $p$-adic integral representation

$$
\left\{\left(\begin{array}{cc}
\hat{M}(\varepsilon) & \hat{M}(\varepsilon) \\
\Delta(\varepsilon) & )^{2}
\end{array}\right.\right.
$$

with an irreducible representation $\left\{\hat{M}(\varepsilon)^{2}\right\}$ is equivalent to

$$
\left\{\left(\begin{array}{ll}
\hat{M}(\varepsilon) & \hat{M}(\varepsilon)
\end{array}\right)^{\prime}\right\}
$$

as a representation over $p$-adic integers. See $\$ 4,6$ in [1]. 
TheOREM 2. If $G(L / K)$ is cyclic, the p-adic representation $\left\{M_{\rho^{\prime}}\left(\eta_{i}\left(\varepsilon^{\prime}\right)\right)\right\}$ of the galois group $G(L / K)$ is equivalent to the direct sum of $\left(\gamma\left(J_{K}\right)-1\right)$-times of the regular representation and the identical representation as a representation over p-adic integers.

Proof. Let $\varepsilon$ be a generator of $G(L / K)$ and $H_{i}$ be the subgroup $\left(\varepsilon^{p^{2}}\right)$ $(i=1,2, \ldots, r)$. We shall prove the theorem by the induction on $G\left(L_{i}^{\prime} K\right) / H_{i}$. If $i=1$, by virtue of lemma 8 , the theorem is true. We assume the theorem on $G\left(L / L_{l_{r-1}}\right)$. Then, since $G$ has only one faithful irreducible $p$-adic integral representation $\left\{\hat{M}(\varepsilon)^{2}\right\}$ and any $p$-adic integral representation of the following type

$$
\left\{\left(\begin{array}{cc}
\hat{M}(\varepsilon) & 0 \\
1(\varepsilon) & \hat{M}(\varepsilon)
\end{array}\right)^{2}\right\}
$$

is equivalent to

$$
\left\{\left(\begin{array}{ll}
\hat{M}(\varepsilon) \quad \hat{M}(\varepsilon) \\
)
\end{array}\right\}\right.
$$

there exists a system of $p$-adic coordinates on $J_{I}$ such that

$$
M_{p}(\eta(\varepsilon))=\left(\begin{array}{ccc}
E_{\because, K-1} \times \hat{M}(\varepsilon) & \\
A(\varepsilon) & E_{\because, K \backslash-1} \times R_{-1}(\Sigma) & \\
b(\varepsilon) & & 1
\end{array}\right)
$$

where $\left\{\hat{M}(\varepsilon)^{2}\right\}$ is the representation of $G(L / K)$ induced by the non-trivial irreducible representation $N\left(\varepsilon^{p^{r-1}}\right)$ of $\left(\varepsilon^{1, r-1}\right)$ and $\left\{R_{r-1}(\varepsilon)^{2}\right\}$ is the regular representation of $G(L / K) / H_{r-1}$.

On the other hand, by virtue of lemma 8 ,

$$
\left\{M_{p}\left(\eta\left(\xi^{1 p^{r-1}}\right)^{*}\right)\right\}
$$

is equivalent to the direct sum of $\left(r\left(J_{k}\right)-1\right) p^{r-1}$-times of the regular representation of $\left(\varepsilon^{p r-1}\right)$ and the identical representation. The latter representation is equivalent to the restriction on $\left(\xi^{t^{r-1}}\right)$ of the direct sum of $\left(\gamma\left(J_{\Lambda}\right)-1\right)$-times of the regular representation of $G(L / K)$ and the identical representation. Therefor, by virtue of lemma 5,7 , we have a system of $p$ adic coordinates on $J_{L}$ such that 


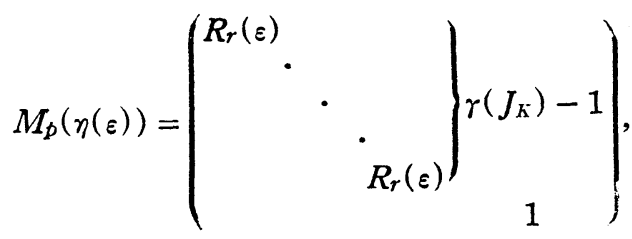

where $\left\{R_{r}(\varepsilon)^{2}\right\}$ is the regular representation of $G(L / K)$.

\section{REFERENCES}

[1] Diederichsen, Über die Ausreduktion ganzzahliger Gruppendarstellungen bei arithmetischer Äquivalenz, Abh. Math. Hamb. Sem. Bd. 13 (1940).

[2] H. Morikawa, Generalized jacobian varieties and separable abelian extensions of function fields, Nagoya Math. Jour. vol. 12 (1957).

[3] Šafarević, On p-extensions, Math. Sbornik N.S. 20 (62), (1947). 